\title{
Long-term feeding of a synthetic diet rich in disaccharides induces hepatic fibrosis in nonalcoholic fatty liver disease in Zucker rats
}

\author{
SHINYA FUKUNISHI ${ }^{1,2}$, HAJIME NISHIO ${ }^{5}$, AKIRA FUKUDA ${ }^{2}$, ATSUSHI TAKESHITA ${ }^{3}$, \\ TOSHIAKI HANAFUSA ${ }^{4}$, KAZUHIDE HIGUCHI ${ }^{2}$ and KOICHI SUZUKI ${ }^{1}$ \\ ${ }^{1}$ Department of Legal Medicine; ${ }^{2}$ Second Department of Internal Medicine; ${ }^{3}$ Department of Pathology; \\ ${ }^{4}$ First Department of Internal Medicine, Osaka Medical College, 2-7 Daigaku-machi, Takatsuki, Osaka, 569-8686; \\ ${ }^{5}$ Department of Legal Medicine, Hyogo Medical College, 1-1 Mukogawa-machi, Nishinomiya, Hyogo, 663-8501, Japan
}

Received September 18, 2009; Accepted November 16, 2009

DOI: 10.3892/ijmm_00000329

\begin{abstract}
Nonalcoholic fatty liver disease (NAFLD) is a chronic liver disease, and is commonly observed in patients with obesity or type 2 diabetes. The pathogenesis of hepatic fibrosis is clinically important to the outcome of NAFLD, however, is not well understood. Since dietary habits are often considered to be responsible for NAFLD, we used a synthetic diet rich in disaccharides $(12.1 \%$ calorie sucrose and lactose), which can be considered for human consumption. We examined the long-term ( 24 weeks) effect of this diet on the liver of Zucker (fa/fa) rats. The synthetic diet-fed Zucker (fa/fa) rats showed hepatic fibrosis during the development of NAFLD with no apparent infiltration of inflammatory cells. They showed significantly elevated hepatic mRNA levels of proinflammatory and profibrogenic cytokines. These findings suggest that excess long-term feeding of a diet similar to the synthetic diet used in our study leads to hepatic fibrosis during the development of NAFLD in patients with obesity or type 2 diabetes. Our results were different from NAFLD accompanied by infiltration of inflammatory cells. However, they also suggest that long-term feeding of this synthetic diet to Zucker (fa/fa) rats is useful for studying the hepatic fibrogenesis during the pathogenesis of NAFLD.
\end{abstract}

Correspondence to: Dr Koichi Suzuki, Department of Legal Medicine, Osaka Medical College, 2-7 Daigaku-machi, Takatsuki, Osaka 569-8686, Japan

E-mail: leg012@art.osaka-med.ac.jp

Abbreviations: NAFLD, nonalcoholic fatty liver disease; TG, triglyceride; NASH, nonalcoholic steatohepatitis; MCD, methioninecholine-deficient; HFD, high-fat diet; ALT, alanine aminotransferase; FFA, free fatty acid; H\&E, hematoxylin and eosin; RT-PCR, reversetranscription polymerase chain reaction; TNF- $\alpha$, tumor necrosis factor- $\alpha$; TGF- $\beta 1$, transforming growth factor- $\beta 1 ; \alpha$-SMA, $\alpha$-smooth muscle actin; HSCs, hepatic stellate cells

Key words: disaccharides, fibrosis, nonalcoholic fatty liver disease, synthetic diet, Zucker rats

\section{Introduction}

Nonalcoholic fatty liver disease (NAFLD) is commonly observed in obese and in type 2 diabetic patients, and is emerging as the most common liver disease in industrialized countries (1-3). NAFLD is a clinical and pathological term that includes a disease spectrum ranging from simple triglyceride (TG) accumulation in hepatocytes to steatosis with inflammation, referred to as nonalcoholic steatohepatitis (NASH) $(4,5)$. The pathogenesis of NASH, which is the more advanced form of NAFLD, has yet to be clearly defined. According to the currently accepted 'two-hit' hypothesis for the progression of NAFLD, hepatic steatosis is a risk factor for NASH and hepatic fibrosis $(6,7)$. However, while there has recently been an interest as to how hepatic steatosis develops in humans, the mechanism of progressive hepatic fibrosis in NAFLD has not been clearly defined $(8,9)$.

Since useful non-invasive techniques for studying hepatic steatosis, steatohepatitis, and hepatic fibrosis in humans are limited, our current knowledge of the pathogenesis is based on data from several animal models. Several animal models for NAFLD, based on the feeding of functional diets such as a methionine-choline-deficient (MCD) diet or high-fat diet (HFD), have been developed $(10,11)$. However, these animal models do not correctly reflect the human pathophysiology of NAFLD, because humans are not able to eat the diet components daily. Since the pathogenesis of human NAFLD is associated with daily diet, studies matched to components of a diet that can be considered for human consumption are necessary to study effective therapies for NAFLD.

Zucker (fa/fa) rats, which are homozygous for a leptin receptor mutation, have a deficiency of leptin signaling, and exhibit hyperphagia, obesity, and type 2 diabetes. It was earlier reported that short-term ( 1 or 3 weeks) feeding of a diet rich in sucrose to Zucker (fa/fa) rats induces NAFLD with no fibrosis and no infiltration of inflammatory cells (12).

Because NAFLD is often associated with obesity and type 2 diabetes in humans, we used Zucker (fa/fa) rats that developed obesity and type 2 diabetes in the present study. According to human lifestyles, on a long-term basis we fed these animals a synthetic diet rich in sucrose and lactose (synthetic 


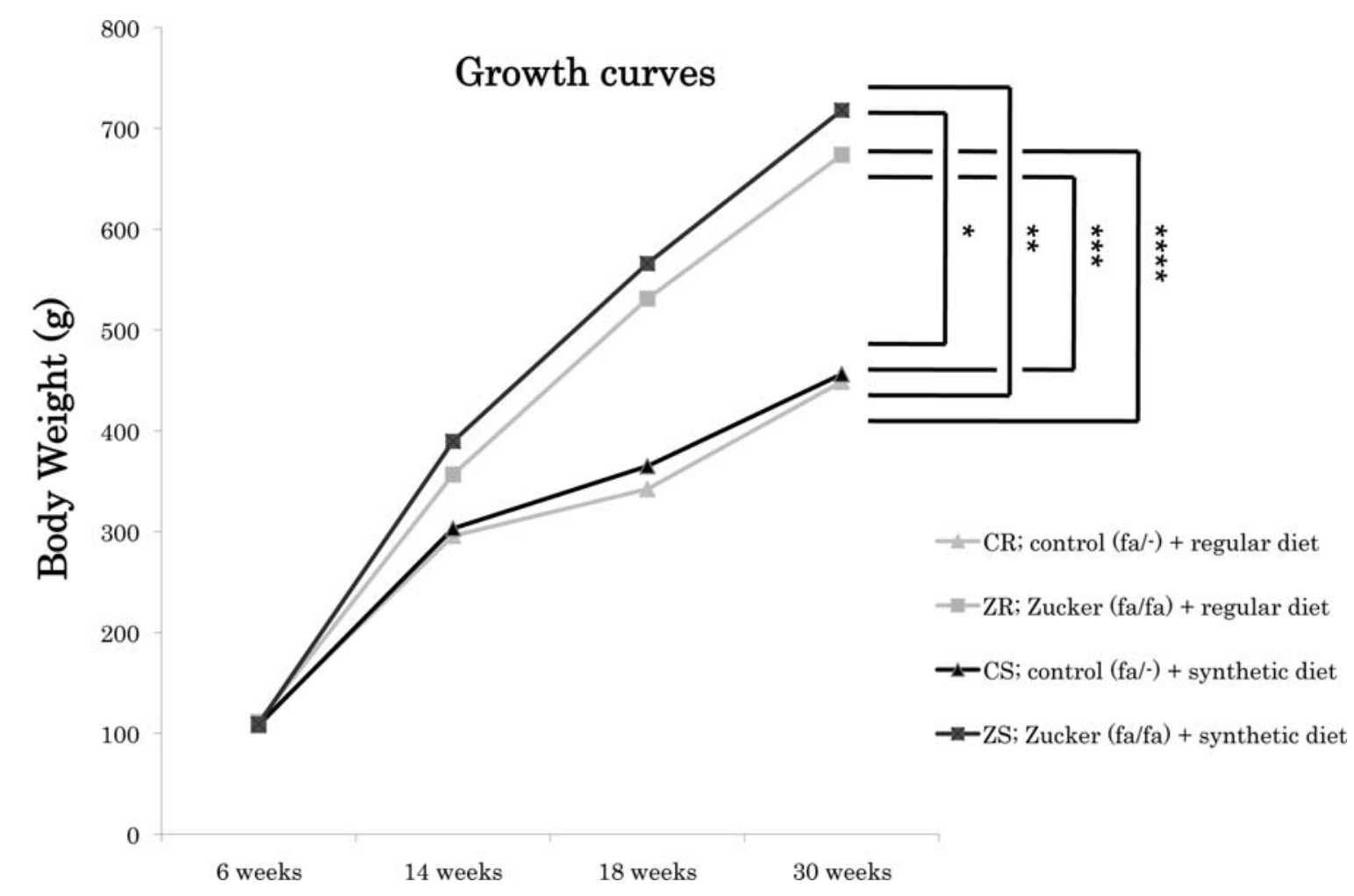

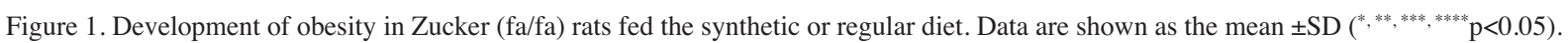

diet), which can be considered for human consumption, and examined the effects of this diet on their liver.

\section{Materials and methods}

Animals. Six-week-old male Zucker (fa/fa) and control (fa/-) rats were purchased from Nippon SLC Co. Ltd. (Shizuoka, Japan). The rats were housed in plastic cages for 24 weeks in an animal room under a 12-h light/12-h dark cycle, controlled temperature $\left(22 \pm 1^{\circ} \mathrm{C}\right)$, and controlled humidity $(50 \pm 10 \%)$, with ad libitum access to food and tap water.

Experimental designs. Rats were fed either a synthetic diet (Clea Japan Inc., Tokyo, Japan) or a regular diet (Funahashi Farm, Chiba, Japan). The calories/g were the same in both diets. The calorie $\%$ (cal\%) of protein, fat, and nitrogen free extract was $20.7,9.8$, and 69.5 cal\%, respectively, in the synthetic diet. In the regular diet, these percentages were 20.7, 12.5 , and $66.8 \mathrm{cal} \%$, respectively, the values thus being similar to those of the synthetic diet. The difference in the 2 diets was the content of disaccharides. The synthetic diet contained $12.1 \mathrm{cal} \%$ (17.4 cal\% to carbohydrate) of sucrose and lactose, whereas the regular diet contained only $0.1 \mathrm{cal} \%$ ( $0.17 \mathrm{cal} \%$ to carbohydrate) of sucrose and lactose. The rats were divided into the following 4 experimental groups: (i) regular diet-fed control (fa/-) rats $(\mathrm{n}=4)$; (ii) synthetic diet-fed control $(\mathrm{fa} /-)$ rats $(n=4)$; (iii) regular diet-fed Zucker (fa/fa) rats $(n=4)$; and (iv) synthetic diet-fed Zucker (fa/fa) rats $(n=4)$. After having been fed the respective diets for 24 weeks, the rats were fasted overnight, and blood and liver samples were taken for further experiments. In addition, to compare the histological findings of previous studies (7), and to confirm the changes in the hepatic tissue during the experimental period, we added the following 2 groups: rats fed the diets for 3 or 12 weeks. All surgical and experimental procedures were performed according to the guidelines for the care and use of animals approved by Osaka Medical College. One set of experiments for a 24-week feeding was carried out three times.

Measurement of plasma biochemical parameters. Plasma alanine aminotransferase (ALT), insulin, glucose, leptin, adiponectin, and free fatty acid (FFA) levels were measured by a local laboratory that performs clinical examinations (SRL Co. Ltd., Tokyo, Japan).

Measurement of hepatic biochemical parameters. Hepatic tissues were homogenized with a Janke and Kunkel Polytron homogenizer (Ultra-Turrax TP18/1051; IKA-Labortechnik, Staufeni, Germany) in buffer, pH 7.4, containing $20 \mathrm{mM}$ Tris $\mathrm{HCl}, 1 \mathrm{mM}$ EGTA, $2 \mathrm{mM}$ EDTA, and protease inhibitor ( $2 \mu \mathrm{g} / \mathrm{ml}$ leupeptin cocktail). Hepatic tissue TG levels were measured by SRL Co. Ltd. (Tokyo, Japan).

Histology. Livers were formalin-fixed, paraffin-embedded and processed for hematoxylin and eosin (H\&E) or Azan staining.

Measurement of mRNA levels in the liver. Total RNA was extracted by using Trizol reagent (Qiagen, Tokyo, Japan) according to the manufacturer's protocol. cDNAs were synthesized from $1 \mu \mathrm{g}$ of the isolated RNA by using a FirstStrand cDNA Synthesis Kit for Reverse-transcription polymerase chain reaction (RT-PCR) (AMV)+ (Roche Diagnostics, Mannheim, Germany). Real-time quantitative RT-PCR was performed for quantitative assessment of mRNA by using a 
Table I. Plasma, hepatic biochemical parameters and liver weight/body weight ratios of the rats fed regular or synthetic diet for 24 weeks.

\begin{tabular}{|c|c|c|c|c|}
\hline & \multicolumn{2}{|c|}{ Regular diet } & \multicolumn{2}{|c|}{ Synthetic diet } \\
\hline & $(\mathrm{fa} /-), \mathrm{n}=4$ & $(\mathrm{fa} / \mathrm{fa}), \mathrm{n}=4$ & $(\mathrm{fa} /-), \mathrm{n}=4$ & $(\mathrm{fa} / \mathrm{fa}), \mathrm{n}=4$ \\
\hline Liver/body ratio (\%) & $2.1 \pm 0.081$ & $2.95 \pm 0.013^{*, * * *}$ & $2.15 \pm 0.17$ & $3.45 \pm 0.041^{*, * *, * * *}$ \\
\hline ALT (IU/l) & $59.82 \pm 8.76$ & $79.0 \pm 17.3^{*}$ & $70.61 \pm 64.16$ & $601.4 \pm 123.18^{*, * * * * * *}$ \\
\hline Leptin (ng/ml) & $6.39 \pm 1.26$ & $98.56 \pm 4.06^{* * * * *}$ & $10.65 \pm 4.54$ & $161.31 \pm 34.21^{*, * *, * * *}$ \\
\hline Adiponectin $(\mu \mathrm{g} / \mathrm{ml})$ & $18.18 \pm 0.43^{* * * *}$ & $18.77 \pm 1.09^{* * * *}$ & $19.42 \pm 2.92^{* * * *}$ & $16.23 \pm 0.57$ \\
\hline $\mathrm{FFA}(\mu \mathrm{EQ} / 1)$ & $674.05 \pm 51.33$ & $873.65 \pm 57.42^{*, * * *}$ & $700.32 \pm 115.06$ & $1452.4 \pm 277.7^{*, * *, * * *}$ \\
\hline Hepatic TG (mg/dl) & $40.25 \pm 24.14$ & $38.62 \pm 8.85$ & $62.94 \pm 30.45$ & $116.38 \pm 13.6^{*, * *, * * *}$ \\
\hline
\end{tabular}

Data are shown as the mean \pm SD. ${ }^{*}$ p $<0.05$ vs. regular diet-fed control (fa/-) rats; ${ }^{* *}$ p $<0.05$ vs. regular diet-fed Zucker (fa/fa) rats; ${ }^{* * *}$ p $<0.05$ vs. synthetic diet-fed control (fa/-) rats; ${ }^{* * * *} \mathrm{p}<0.05$ vs. synthetic diet-fed Zucker (fa/fa) rats.

LightCycler (Roche Diagnostics) according to the manufacturer's protocol. The mRNAs examined and the sequences of the primers used were the following, TNF- $\alpha$ (TNFF3, 5'-CAAA ACTCGAGTGACAAGCCCGT-3'; TNF-R3, 5'-GAGATCC ATGCCATTGGC-3'); TGF-ß1 (TGF-F2, 5'-TGCTTCAGCT CCACAGAGAA-3'; TGF-R2, 5'-TACTGTGTGTCCAGGC TCCA-3'); $\alpha$-SMA (SMA-F1, 5'-TGTGCTGGACTCTGGA GATG-3'; SMA-R1, 5'-TCCAGAGCGACATAGCACAG-3'); and type 1 collagen $\alpha 1$ (Col-F1, 5'-GAGAGCATGACCGAT GGATT-3'; Col-R1, 5'-TTGAGGTTGCCAGTCTGTTG-3'). The relative levels of the target mRNAs were normalized to the corresponding levels of GADPH mRNA (GADPH-F, 5'-TGAACGGGAAGCTCACTGG-3'; GADPH-R, 5'-TCCA CCACCCTGTTGCTGTA-3') in the same cDNA sample by using a standard curve method recommended in the LightCycler Software Ver 3.5 (Roche Diagnostics). All PCR reactions and analyses were performed according to the manufacturer's protocols (Roche Diagnostics).

Statistical analysis. All data were expressed as the mean $\pm \mathrm{SD}$. The significance of differences among values was analyzed by using the unpaired Student's t-test. Differences were considered significant at $\mathrm{p}<0.05$.

\section{Results}

According to our view that the MCD diet, HFD, and diet reported by Novikoff (12) do not correspond to the diets imposed by human lifestyles, we fed a synthetic diet that can be considered for human consumption, to obese, diabetic Zucker (fa/fa) rats. In most major models of NASH, such as MCD diet-fed rats, the animals lose body weight. However, the Zucker (fa/fa) rats given the dietary protocol in this study gained body weight and developed hepatic steatosis with fibrosis. Zucker (fa/fa) and control (fa/-) groups of 6- and 30 -week-old male rats were fed the regular or synthetic diet for 24 weeks. Thus, 4 groups of rats with 2 different diets and of 2 different genetic backgrounds were compared for physiological, and biochemical parameters related to the development of NAFLD. These 4 groups were abbreviated as follows: ZS, synthetic diet-fed Zucker (fa/fa) rat group $(\mathrm{n}=4)$; ZR, regular diet-fed Zucker (fa/fa) rat group ( $n=4)$; CS, synthetic diet-fed control (fa/-) rat group $(\mathrm{n}=4)$; $\mathrm{CR}$, regular diet-fed control $(\mathrm{fa} /-)$ rat group $(n=4)$.

Body weight and liver/body weight ratio. Significant differences in body weight were observed between the 2 groups on the same diet but having a different genetic background (ZS vs. CS and ZR vs. CR) at 24 weeks ( $<<0.05$, Fig. 1). No significant differences were observed between the 2 groups with different diets on the same genetic background (ZS vs. ZR and CS vs. CR) (Fig. 1). The liver/body weight ratio was significantly increased in ZS compared with the ratio for CS or CR $(\mathrm{p}<0.05$, Table I).

Metabolic dysfunction in Zucker (fa/fa) rats. ZS showed the significantly highest levels of plasma insulin among all groups at 24 weeks $(\mathrm{p}<0.05$, Fig. $2 \mathrm{~A})$. The levels in $\mathrm{ZS}$ rats increased at 24 weeks by more than twice that at 12 weeks $(\mathrm{p}<0.05$, Fig. 2A). On the other hand, ZS animals showed the highest level of plasma glucose among all groups at 24 weeks, but the levels were found not to differ significantly among all groups or between 12 and 24 weeks within each group (Fig. 2B).

Liver damage in Zucker ( $f a / f a)$ rats. To examine whether the synthetic diet induced liver damage, we quantified the plasma ALT level. The levels in the ZS group were $~ 8-10$ times significantly higher than those in the other groups at 24 weeks $(\mathrm{p}<0.05$, Table I). The accumulation of TG within the liver is a hallmark of NAFLD. Therefore, hepatic TG levels were compared among the groups at 24 weeks and found to be 2-3 times significantly higher in ZS than in the other groups at 24 weeks $(\mathrm{p}<0.05$, Table I). In addition, plasma free fatty acid (FFA) levels were significantly higher in ZS and ZR than in CS or CR $(\mathrm{p}<0.05$, Table I). In particular, ZS showed $\sim 2$ times higher levels of plasma FFA than ZR ( $<<0.05$, Table I). The presence of excess plasma FFA has been validated in animal models of NASH $(13,14)$.

Next, plasma leptin levels were evaluated for each group because leptin regulates appetite and energy expenditure and contributes, to some extent, to hepatic fibrosis in the development of NAFLD (15-17). Remarkably high levels of leptin were observed in ZS and ZR, being $\sim 15$ times higher than those in CS and CR (Table I). The plasma adiponectin 

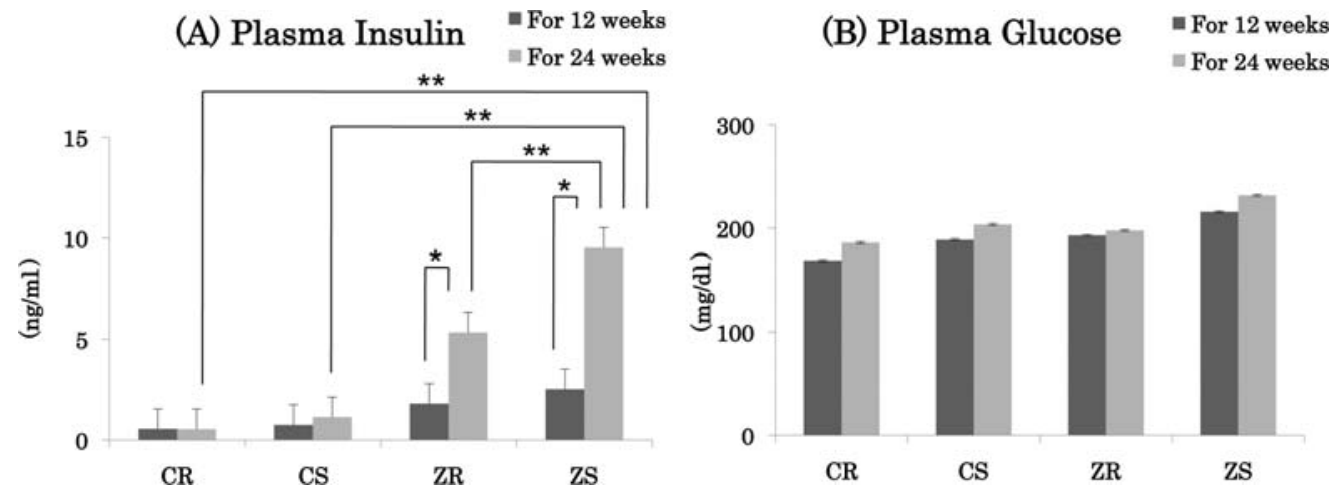

Figure 2. Effects of feeding the diets for 12 or 24 weeks on plasma activities of insulin resistance-associated parameters. (A) plasma glucose in control (fa/-) rats and in Zucker (fa/fa) rats fed the regular diet or synthetic diet for 12 or 24 weeks. (B) Plasma insulin in control (fa/-) rats and in Zucker (fa/fa) rats fed the regular diet or synthetic diet for 12 or 24 weeks $(*, * * 0<0.05)$.

regular diet for 24 weeks
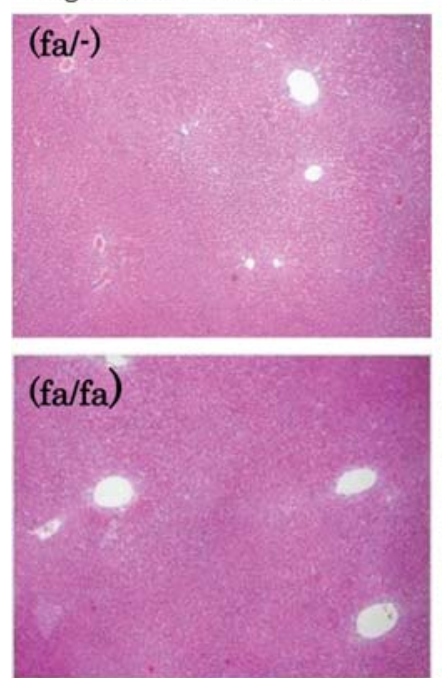

synthetic diet for 3 weeks
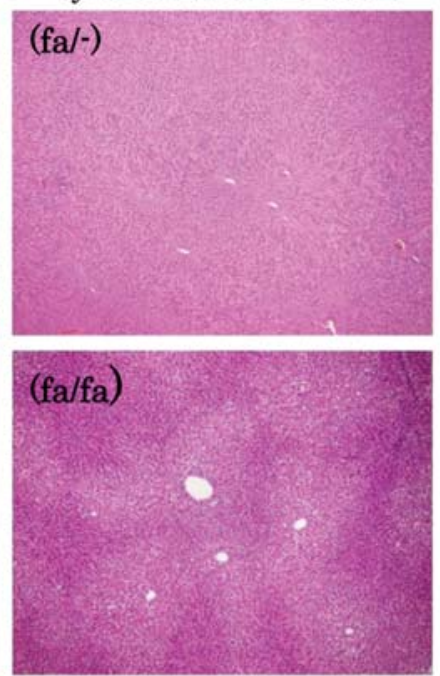

synthetic diet for 12 weeks
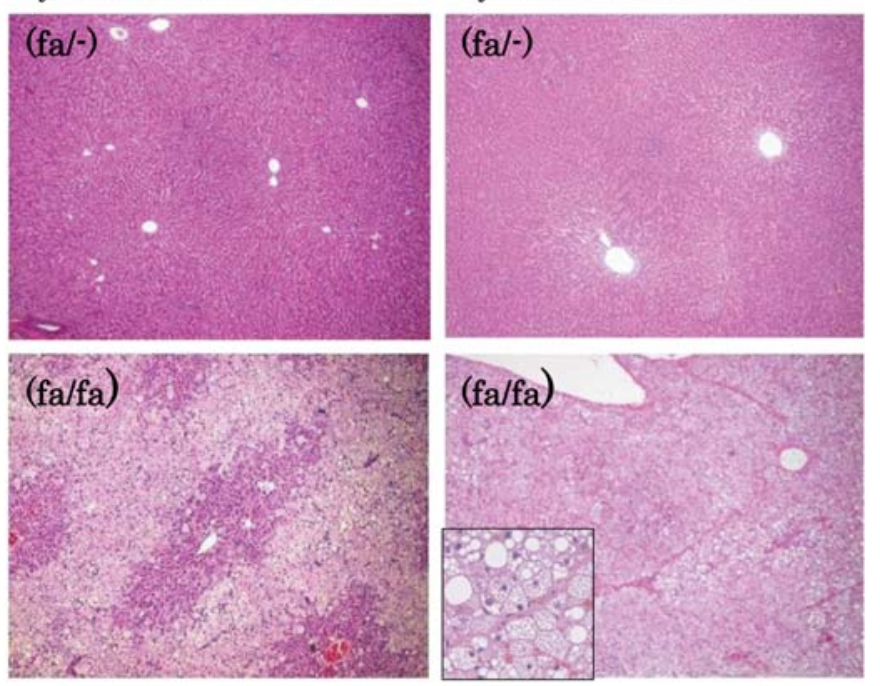

\section{HE staining}

Figure 3. Effects of feeding the synthetic diet on Zucker (fa/fa) rats. Most small fatty droplets were observed in the liver of Zucker (fa/fa) rats fed the synthetic diet for 24 weeks. H\&E staining (original magnification, x100).

level, a hallmark of NAFLD, is decreased in patients with obesity or type 2 diabetes, and adiponectin has a preventive effect on hepatic fibrosis $(18,19)$. The level in the ZS showed the lowest level among all groups (Table I).

Histology. Biochemical examinations of blood and liver suggested liver damage and steatosis. To compare our data with those of Novikoff (12), we examined the liver histology. Large amounts of fatty droplets were observed only in the ZS group at 24 weeks (Fig. 3). Furthermore, the liver of the ZS rats showed remarkable fibrosis in perivenous and periportal areas, which were bridged in some areas (Fig. 4). A striking finding was that no apparent infiltration of inflammatory cells was found, despite the appearance of hepatic fibrosis.

Expression of hepatic mRNAs in Zucker (falfa) rats. Such histological findings suggest the expression of cytokines involved in inflammation and fibrosis. Hepatic tumor necrosis factor (TNF)- $\alpha$ and transforming growth factor (TGF)- $\beta 1$
mRNAs were examined and found to be relatively increased in the ZS rats (Fig. 5A and B), the latter of the expressed products is known to activate hepatic stellate cells (HSCs) (20). Activation of the cells were then confirmed by increased expression of $\alpha$-smooth muscle actin (SMA, Fig. 5C), a marker of $\mathrm{HSC}$ activation, which resulted in the accumulation of type 1 collagen, a molecule that plays a central role of fibrosis in the liver (Fig. 5D) $(21,22)$.

\section{Discussion}

To evaluate the pathogenesis of NAFLD under the dietary components that can be considered for human consumption, we fed a synthetic diet rich in sucrose and lactose, long-term, to Zucker (fa/fa) rats, which developed obesity and type 2 diabetes. We found that our protocol led to the development of NAFLD in Zucker (fa/fa) rats, as confirmed by histological findings. Elevation of plasma ALT levels and increased accumulation of hepatic TG indicated liver damage and 

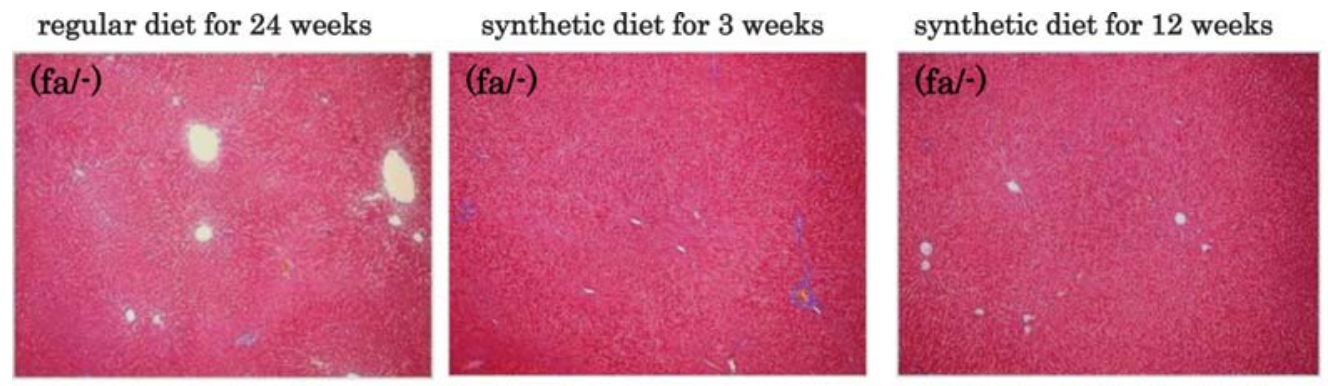

synthetic diet for 24 weeks
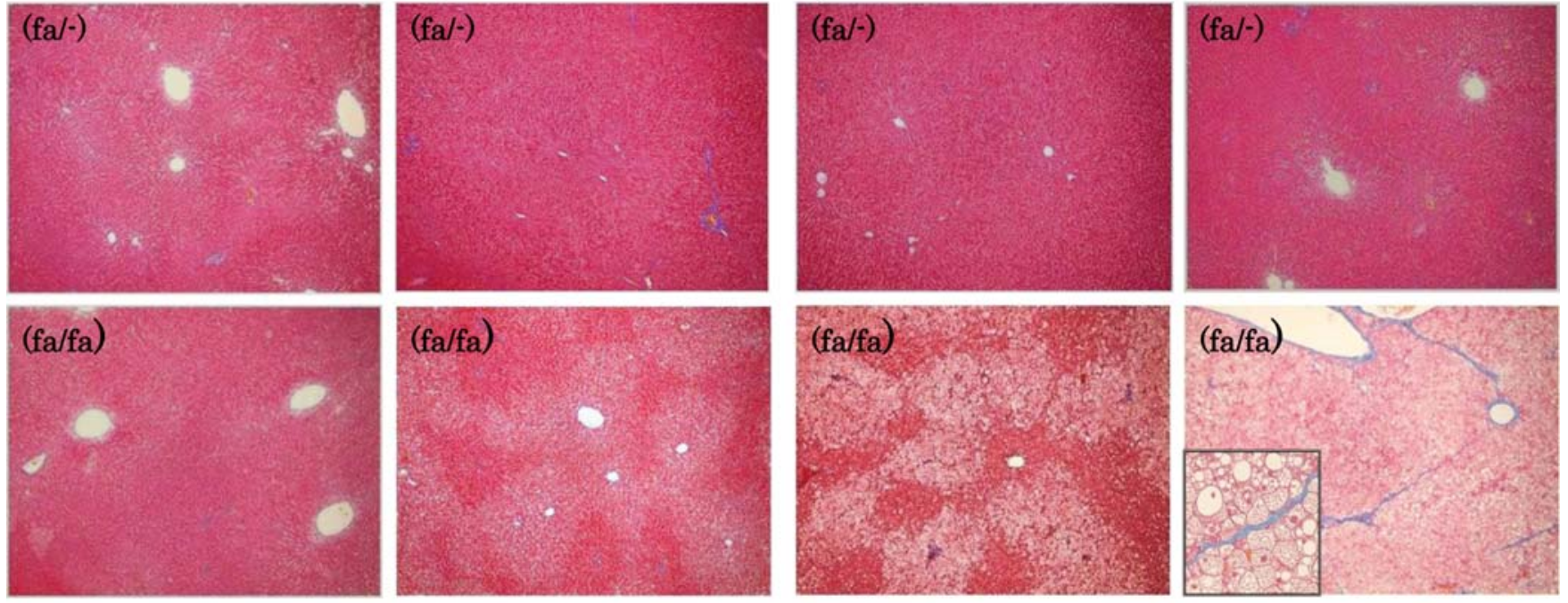

\section{Azan staining}

Figure 4. Effects on Zucker (fa/fa) rats fed a synthetic diet for 24 weeks. Remarkable fibrosis is observed in the liver of the rats fed the synthetic diet for 24 weeks. Azan staining (original magnification, x100).

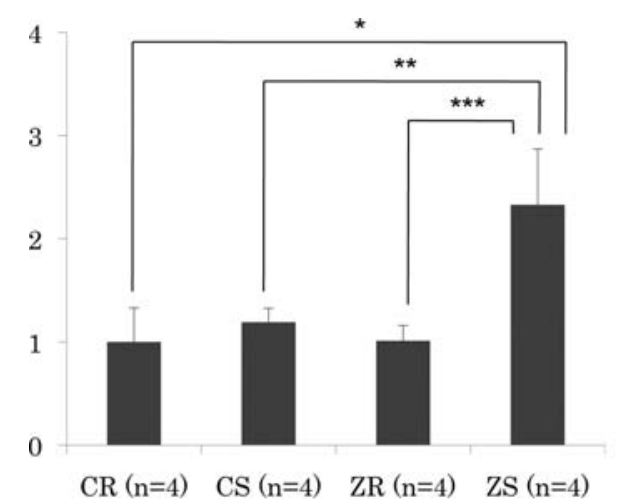

(A) Relative TNF-a mRNA expression

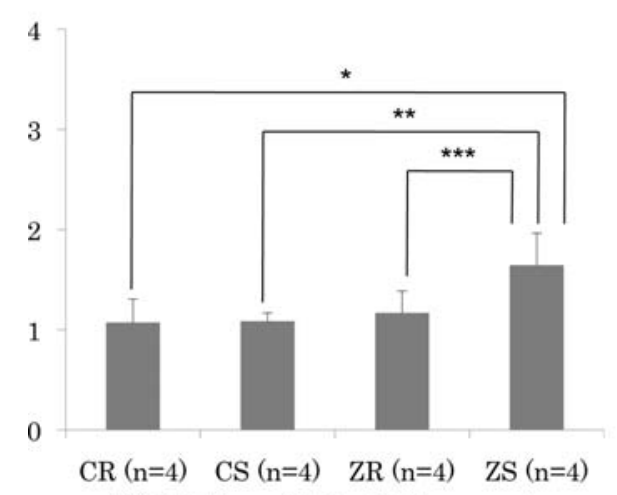

(C) Relative $\alpha$-SMA mRNA expression

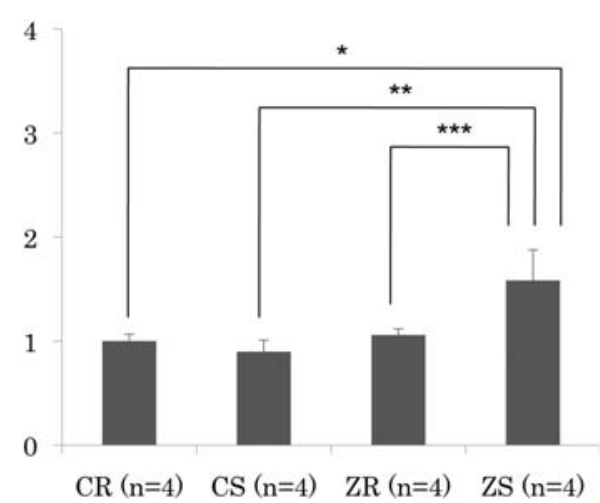

(B) Relative TGF-81 mRNA expression

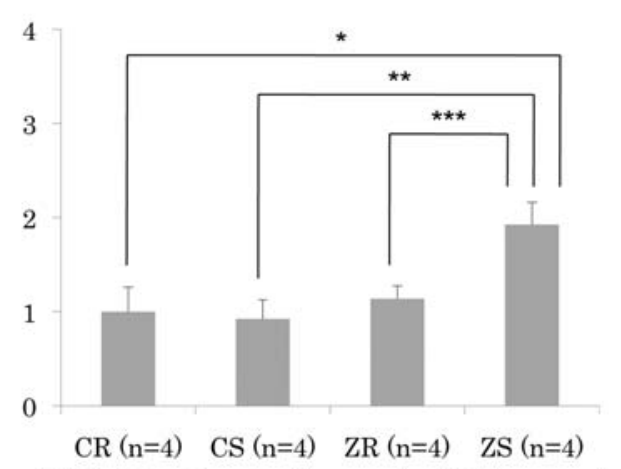

(D) Relative type 1 collagen a1 mRNA expression

Figure 5. (A) Expression of TNF- $\alpha$; (B) Expression of TGF-ß1; (C) Expression of $\alpha$-SMA; (D) Expression of type 1 collagen $\alpha 1$ in the liver of the synthetic diet-fed Zucker (fa/fa) rats. Quantitative RT-PCR reveals that mRNA levels of TNF- $\alpha$, TGF- $\beta 1, \alpha$-SMA and type 1 collagen $\alpha 1$ are significantly increased in synthetic diet-fed Zucker (fa/fa) rats. Data are shown as the mean $\pm \operatorname{SD}\left({ }^{*, * * * * * *} \mathrm{p}<0.05\right)$.

steatosis in the rats. In addition, the decreased level of plasma adiponectin was also associated with hepatic vulnerability. Furthermore, the observed elevated plasma glucose and insulin levels indicated the induction of insulin resistance in the rats.
The results of biochemical examinations showed that our protocol induced NAFLD with metabolic abnormalities in the rats, as is the case in human NAFLD. In addition to these metabolic abnormalities, our protocol stimulated the expression 
of profibrogenic mRNAs such as those of TNF- $\alpha$, TGF- $\beta 1$, $\alpha$-SMA, and type 1 collagen $\alpha 1$ in the liver. The increased expression of these hepatic mRNAs, corresponded to the histological findings made on the liver of the synthetic dietfed Zucker (fa/fa) rats.

Interestingly, our protocol induced hepatic fibrosis with no apparent infiltration of inflammatory cells in the synthetic diet-fed Zucker (fa/fa) rats, whereas no fibrosis was observed in the regular diet-fed Zucker (fa/fa) rats. Thus, long-term feeding of the synthetic diet induced NAFLD with fibrosis in the Zucker (fa/fa) rats. In addition, elevation of plasma leptin levels also may be associated with the development of hepatic fibrosis in these synthetic diet-fed Zucker (fa/fa) rats (15-17).

In this study, it was important that our protocol increased the body weight of Zucker (fa/fa) rats, as such an increase is observed in human NAFLD. Since histological features of the liver in the MCD diet model are similar to those of human NAFLD, a number of significant studies have been performed on the MCD diet model (23). However, body weight in experimental rats and mice decreases when the animals are given the MCD diet; whereas a decrease in body weight is rarely observed in actual human NAFLD $(24,25)$. Therefore, we conclude that the MCD diet does not coincide with the development of NAFLD, at least in part.

The histological findings in our study might provide insight, which the MCD diet model has not shown yet, into the relationship between hepatic fibrosis and infiltration of inflammatory cells with respect to the development of NAFLD.

The Zucker (fa/fa) rat lacks the leptin signaling due to mutated leptin receptors. Although this lack is rarely seen in obese patients, Zucker (fa/fa) rats reflect metabolic abnormalities found in humans (26). Leptin is involved in immune responses and to some extent in fibrosis in the liver $(27,28)$. The increase in the plasma leptin level in ZS rats observed in this study may be associated with the development of hepatic fibrosis. This association leads us to the conclusion that obesity itself is a risk factor for hepatic fibrosis.

Dietary habits seem to be deeply involved in the development of NAFLD. Novikoff reported an experimental model diet containing a high content of disaccharides such as sucrose (12). Short-term (1-3 weeks) consumption of this diet caused Zucker (fa/fa) rats to develop hepatic steatosis with no apparent infiltration of inflammatory cells nor fibrosis. Hepatic steatosis in this model must be due to such a high content of sucrose (85.7 cal\% to carbohydrate) in the diet, an unusual amount in human daily diets (12). On the other hand, the ratio of disaccharides ( $17.4 \mathrm{cal} \%$ to carbohydrate) contained in the synthetic diet used in our study is common in the human daily diet, compared to the MCD diet and the diet reported by Novikoff. Disaccharides such as sucrose and lactose raise the blood glucose level rapidly. In the setting of energy excess caused by the excessive intake of disaccharides, the glucose is converted to fatty acids in hepatocytes. As a result, these cells use these fatty acids to synthesize TG. Therefore, long-term feeding of the diet to Zucker (fa/fa) rats may result in an energy excess, and result in the development of NAFLD.

In conclusion, we showed metabolic abnormalities and obesity in Zucker (fa/fa) rats fed a long-term, synthetic diet containing disaccharides. Our results suggest that daily intake of products containing disaccharides induces NAFLD in type 2 diabetes and obese patients. Although some issues remain to be elucidated, the synthetic diet-fed Zucker (fa/fa) rats will assist in advancing our understanding of the relationship between hepatic fibrosis and infiltration by inflammatory cells with respect to the development of NAFLD.

\section{Acknowledgements}

This work was supported by a grant from the Ministry of Education, Culture, Sports, Science, and Technology of Japan to H. Nishio (No. 18590645).

\section{References}

1. Fong DG, Nehra V, Lindor KD and Buchman AL: Metabolic and nutritional considerations in nonalcoholic fatty liver. Hepatology 32: 3-10, 2000.

2. Harrison SA and Diehl AM: Fat and the liver - a molecular overview. Semin Gastrointest Dis 13: 3-16, 2002.

3. Youssef W and McCullough AJ: Diabetes mellitus, obesity, and hepatic steatosis. Semin Gastrointest Dis 13: 17-30, 2002.

4. Matteoni CA, Younossi ZM, Gramlich T, Boparai N, Liu YC and McCullough AJ: Nonalcoholic fatty liver disease: a spectrum of clinical and pathological severity. Gastroenterology 116: 1413-1419, 1999.

5. Neuschwander-Tetri BA and Caldwell SH: Nonalcoholic steatohepatitis: summary of an AASLD single topic conference. Hepatology 37: 1202-1219, 2003.

6. Day CP and James OFW: Steatohepatitis: A tale of two 'hits'? Gastroenterology 114: 842-845, 1998.

7. Koteish A and Diehl AM: Animal models of steatohepatitis. Best Pract Res Clin Gastroenterol 16: 679-690, 2002.

8. Angulo P: Nonalcoholic fatty liver disease. N Engl J Med 346: 1221-1231, 2002.

9. Clark JM, Brancati FL and Diehl AM: Nonalcoholic fatty liver disease. Gastroenterology 122: 1649-1657, 2002.

10. Chen Y, Hozawa S, Sawamura S, Sato S, Fukuyama N, Tsuji C, Mine T, Okada Y, Tanino R, Ogushi Y and Nakazawa H: Deficiency of inducible nitric oxide synthase exacerbates hepatic fibrosis in mice fed high-fat diet. Biochem Biophys Res Commun 326: 45-51, 2004.

11. Sundaram SS, Whitington PF and Green RM: Steatohepatitis develops rapidly in transgenic mice overexpressing Abcb11 and fed a methionine-choline-deficient diet. Am J Physiol Gastrointest Liver Physiol 288: G1321-G1327, 2005.

12. Novikoff PM: Fatty liver induced in Zucker 'fatty' (ff) rats by a semisynthetic diet rich in sucrose. Pro Natl Acad Sci USA 74: 3038-3042, 1977.

13. Hensley K, Kotake Y, Sang H, Pye QN, Wallis GL, Kolker LM, Tabatabaie T, Stewart CA, Konishi Y, Nakae D and Floyd RA: Dietary choline restriction causes complex I dysfunction and increased $\mathrm{H}_{2} \mathrm{O}_{2}$ generation in liver mitochondria. Caricinogenesis 21: $983-989,2000$.

14. Yang SQ, Zhu H, Li Y, Lin HZ, Gabrielson K, Trush MA and Diehl AM: Mitochondrial adaptations to obesity-related oxidant stress. Arch Biochem Bipohys 378: 259-268, 2000.

15. Potter JJ, Womack L, Mezey E and Anania FA: Transdifferentiation of rat hepatic stellate cells results in leptin expression. Biochem Biophys Res Commun 244: 178-182, 1998.

16. Saxena NK, Ikeda K, Rockey DC, Friedman SL and Anania FA: Leptin in hepatic fibrosis: evidence for increased collagen production in stellate cells and lean littermates of ob/ob mice. Hepatology 35: 762-771, 2002.

17. Zhang Y, Proenca R, Maffei M, Barone M, Leopold L and Friedman JM: Positional cloning of the mouse obese gene and its human homologue. Nature 372: 425-432, 1994.

18. Halleux CM, Takahashi M, Delporte ML, Detry R, Funahashi T, Matsuzawa Y and Brichard SM: Secretion of adiponectin and regulation of apM1 gene expression in human visceral adipose tissue. Biochem Biophys Res Commun 288: 1102-1107, 2001.

19. Kamada Y, Tamura S, Kiso S, Matsumoto H, Saji Y, Yoshida Y, Fukui K, Maeda N, Nishizawa H, Nagaretani H, Okamoto Y, Kihara S, Miyagawa J, Shimomura Y, Funahashi T and Matsuzawa Y: Enhanced carbon tetrachloride-induced liver fibrosis in mice lacking adiponectin. Gastroenterology 125 : 1796-1807, 2003. 
20. Matsuoka M and Tsukamoto H: Stimulation of hepatic lipocyte collagen production by Kupffer cell-derived transforming growth factor B: implication for a pathogenetic role in alcoholic liver fibrogenesis. Hepatology 11: 599-605, 1990.

21. Enzan H, Himeno H, Iwamura S, Saibara T, Onishi S and Yamamoto Y: Immunohistochemical identification of Ito cells and their myofibroblastic transformation in adult human liver. Virchows Archiv 424: 249-256, 1994.

22. Friedman SL: The cellular basis of hepatic fibrosis-mechanism and treatment strategies. N Engl J Med 328: 1828-1835, 1993.

23. Green RM: NASH: hepatic metabolism and not simply the metabolic syndrome. Hepatology 38: 14-17, 2003

24. Marchesini G, Bugianesi E, Forlani G, Cerrelli F, Lenzi M Manini R, Natale S, Vanni E, Villanova N, Melchionda N and Rizzetto M: Nonalcoholic fatty liver, steatohepatitis, and the metabolic syndrome. Hepatology 37: 917-923, 2003.
25. Rinella ME and Green RM: The methionine-choline deficient dietary model of steatohepatitis dose not exhibit insulin resistance. J Hepatol 40: 47-51, 2004.

26. Paracchini V, Pedotti $P$ and Taioli E: Genetics of leptin and obesity: a HuGE review. Am J Epidemiol 162: 101-114, 2005.

27. Faggioni R, Jones-Carson J, Reed DA, Dinarello CA, Feingold KR, Grunfeld $C$ and Fantuzzi G: Leptin-deficient (ob/ob) mice are protected from $\mathrm{T}$ cell-mediated hepatotoxicity: role of tumor necrosis factor $\alpha$ and IL-18. Proc Natl Acad Sci USA 97: 2367-2372, 2000.

28. Lang T, Ikegima K, Yoshikawa M, Enomoto N, Iijima K, Kitamura T, Takei Y and Sato N: Leptin facilitates proliferation of hepatic stellate cells through up-regulation of platelet-derived growth factor receptor. Biochem Biophys Res Commun 323: 1091-1095, 2004. 